\title{
PATOLOGIAS MAIS FREQUENTES E PERFIL DE USUÁRIOS DE UMA UNIDADE BÁSICA DE SAÚDE
}

\author{
MOST FREQUENT PATHOLOGIES AND USER PROFILE OF A BASIC HEALTH UNIT
}

\author{
Thayná de Oliveira ${ }^{1}$ \\ Marina Werner ${ }^{2}$ \\ Diane Favretto ${ }^{3}$
}

RESUMO: A atenção básica à saúde (ABS) é um conjunto de ações de saúde envolvendo a promoção, prevenção, diagnóstico, tratamento e reabilitação da população. Conhecer o perfil e necessidades da demanda das Unidades Básicas de Saúde (UBSs) é uma das estratégias à fim de proporcionar melhorias em saúde. Definindo o perfil dos usuários e doenças mais prevalentes, é possível criar uma agenda de prioridades para intervir nestes problemas. Objetivo: analisar o perfil de usuários atendidos pelo setor de nutrição de uma UBS do MeioOeste catarinense entre os anos de 2019 e 2020 e identificar as principais doenças para a procura pela Unidade. Métodos: trata-se de um estudo retrospectivo, documental de abordagem quantitativa, com a análise de prontuários. Foram selecionados 284 prontuários para as análises. Resultados: a amostra fora predominantemente do sexo feminino $(64,8 \%)$, com média de idade de $4 \mathrm{I}$ e 60 anos $(5 \mathrm{I}, 8 \%)$. O estado nutricional que prevaleceu nos adultos foi de obesidade grau I e em idosos foi a eutrofia. A obesidade, diabetes mellitus tipo 2 e as dislipidemias foram as patologias prevalentes com $2 \mathrm{I}, \mathrm{I} \%, \mathrm{I} 3,7 \%$ e $12 \%$, respectivamente. Conclusão: $O$ estudo auxiliou para uma melhor caracterização dos usuários da UBS, fornecendo maiores informações, as quais podem ser utilizadas à um futuro planejamento e aperfeiçoamento de programas no âmbito da saúde, principalmente em relação às ações de alimentação e nutrição. Ficando evidente também a importância do nutricionista na $\mathrm{AB}$, em que este tem papel fundamental na prevenção de doenças e controle do estado nutricional dos usuários.

Palavras chave: Unidade Básica de Saúde. Atenção Primária à Saúde. Perfil de saúde.

ABSTRACT: Primary health care (PHC) is a set of health actions involving promotion, prevention, diagnosis, treatment and rehabilitation of the population. Knowing the profile and needs of the demand of Basic Health Units (BHUs) is one of the strategies in order to provide improvements in health. By defining the profile of users and most prevalent diseases, it is possible to create a priority schedule to intervene in these problems. Objective: to analyze the profile of users assisted by the nutrition sector of a BHU in the Midwest of Santa Catarina between 2019 and 2020 and identify the main diseases for the search for the Unit. Methods: this is a retrospective, documentary study of quantitative approach, with

\footnotetext{
' Acadêmica do curso de Nutrição da Universidade do Oeste de Santa Catarina-UNOESC, campus de VideiraSC. E-mail: oliveira_thay@yahoo.com.br ORCID: https://orcid.org/oooo-ooo2-8202-9899.

${ }^{2}$ Docente no curso de Nutrição da Universidade do Oeste de Santa Catarina-UNOESC, campus de VideiraSC. E-mail: marinacwerner@gmail.com ORCID: https://orcid.org/oooo-ooor-6821-2729.

3 Especialista em Nutrição Clínica e Oncológica. E-mail: dianefavretto@gmail.com. ORCID: https://orcid.org/oooo-ooo2-9775-8197
} 
the analysis of medical records. A total of 284 medical records were selected for the analyses. Results: the sample was predominantly female (64.8\%), with a mean age of $4 \mathrm{I}$ and 60 years (51.8\%). The nutritional status that prevailed in adults was grade I obesity and in the elderly was eutrophy. Obesity, type 2 diabetes mellitus and dyslipidemias were the prevalent pathologies with 21.1\%, $13.7 \%$ and $12 \%$, respectively. Conclusion: The study helped to better characterize BHU users, providing more information, which can be used for future planning and improvement of health programs, especially in relation to food and nutrition actions. It is also evident the importance of the nutritionist in PC, in which it plays a fundamental role in the prevention of diseases and control of the nutritional status of users.

Kew- words: Health Centers. Primary Health Care. Health Profile.

\section{INTRODUÇÃO}

A Estratégia Saúde da Família (ESF), é uma das principais estratégias organizacionais da Atenção Primária à Saúde (APS), que é um elemento chave para estruturar Sistemas Nacionais de Saúde, sendo esta, a porta de entrada às redes de atenção à saúde, que busca garantir o acesso igualitário para todos, com suas ações e serviços de forma ordenada (FERREIRA et al., 2017).

A atenção básica à saúde (ABS) é um conjunto de ações de saúde envolvendo a promoção, prevenção, diagnóstico, tratamento e reabilitação no âmbito individual e coletivo da população. Assim, os indivíduos que vivem na área de abrangência de algum Centro de Saúde, podem procurar a Unidade Básica de Saúde (UBS) por variadas causas, como: diagnóstico e tratamento de alguma patologia; prevenção de doenças através da imunização; exames de rastreamento; planejamento familiar; tratamento das doenças crônicas com o recebimento regular de medicamentos; dentre outros motivos (PIMENTEL et al., 20II).

É importante o diagnóstico das principais necessidades e patologias dos indivíduos, para que o sistema de ABS possa solucionar os problemas mais prevalentes dos usuários delimitados pelo Centro de Saúde. Embora seja ampla a variedade desses problemas, existem os mais frequentes, responsáveis por basicamente metade de toda a demanda trazida pelas pessoas. Assim, definindo o perfil dos usuários e doenças mais prevalentes, é possível criar uma lista de alternativas e prioridades para intervir nestes problemas (PIMENTEL et al., 20II). 
Por isso, o objetivo desse estudo é caracterizar o perfil de usuários do SUS de uma UBS e as principais patologias que os levam à procura dos serviços, para o planejamento de futuras estratégias de saúde de forma mais eficaz.

\section{METODOLOGIA}

Trata-se de um estudo retrospectivo, documental de abordagem quantitativa, com base na análise de prontuários. Foi realizado em uma Unidade Básica de Saúde do município de Joaçaba, Santa Catarina.

Os dados foram coletados de prontuários dos anos de 2019 e 2020, escolhidos aleatoriamente. Como critério de inclusão, foram selecionados prontuários de pacientes de 30 anos ou mais, com informações claras e completas, de ambos os sexos; e foram excluídos prontuários de pacientes com idade inferior a 30 anos, cujas informações não foram claras ou faltavam informações.

As anamneses e diagnósticos nutricionais contidos nos prontuários foram realizados durante os atendimentos da nutricionista da Unidade entre os anos de 2019 e 2020. Para a organização dos dados foi elaborada uma planilha utilizando o programa Microsoft Excel.

Após a análise de prontuários, foi demonstrado os resultados por meio de recursos informáticos, com o aplicativo Microsoft Excel para elaboração de tabelas/gráficos e Microsoft Word para descrever as conclusões obtidas.

Este artigo foi submetido e aprovado pelo Comitê de Ética e Pesquisa (CEP) da Universidade do Oeste de Santa Catarina - UNOESC, sob parecer número 4.868.467.

\section{RESULTADOS E DISCUSSÃO}

No período de estudo, foram avaliados um total de 415 prontuários, sendo que destes foram selecionados 284 que se encaixaram nos critérios de inclusão, sendo de ambos os sexos $158(55,6 \%)$ do ano de 2019 e $126(44,4 \%)$ do ano de 2020 , conforme tabela I.

Tabela I - Período de coleta de dados conforme ano e sexo.

$\begin{array}{ccc}\substack{\text { Sexo Feminino } \\(n=184)} & \text { Sexo Masculino } & \text { Total } \\ (n=100) & (n=284)\end{array}$




\begin{tabular}{cccc}
\hline Ano & & & \\
\hline 2019 & 97 & 61 & 158 \\
\hline 2020 & 87 & 39 & 126 \\
\hline
\end{tabular}

Fonte: as autoras.

Os resultados mostraram predominância no sexo feminino $(n=184)$ correspondendo a um percentual de 64,8\%. Dados quais corroboram com o trabalho de Pimentel et. al (201I), em que houve predominância ao atendimento à mulher. Isto pode ser devido uma possível percepção diferenciada das mulheres ao processo saúde-doença, revelando maior interesse destas a buscar cuidados à saúde. Outro fator que deve ser mencionado é a procura das mulheres pelo planejamento familiar, isto também contribui para a maior prevalência do sexo feminino nos Centros de Saúde (PIMENTEL et al., 2oir).

A faixa etária predominante foi de $4 \mathrm{I}$ a 60 anos $(51,8 \%)$ em ambos os sexos, sendo $\mathrm{n}=$ $99(67,3 \%)$ do sexo feminino e $n=48(32,7 \%)$ do sexo masculino, conforme tabela 2.

Tabela 2 - Caracterização dos usuários segundo o sexo e idade

Feminino Masculino Total

Idade $\quad(n=184) \quad(n=100) \quad(n=284)$

$\begin{array}{llll}30-40 & 36 & 25 & 61\end{array}$

\begin{tabular}{cccc}
\hline $\mathbf{4} \mathbf{I}-\mathbf{6 0}$ & 99 & 48 & $\mathrm{I} 47$ \\
\hline$>60$ & 49 & 27 & 76 \\
\hline
\end{tabular}

Fonte: as autoras.

Com base na classificação segundo o índice de massa corporal (IMC), o IMC menor que 18,5 considera-se "magro ou baixo peso", entre 18,5 e 24,9 "normal ou eutrófico", 25 e 29,9 “sobrepeso ou pré-obeso", 30 e 34,9 "obesidade grau I", 30 e 39,9 "obesidade grau II" e $\geq$ 40,0 “obesidade grave ou obesidade grau III" (ABESO, 2016), verificou-se neste estudo, que o estado nutricional prevalente da amostra da população adulta total, foi de obesidade grau I, em 77 indivíduos $(37,0 \%)$, em que, 51 (66,2\%) eram mulheres e $26(33,8 \%)$ eram homens. 
Contudo cabe mencionar que a eutrofia segue a obesidade grau I, em 38 (I8,3\%) usuários, sendo 22 mulheres $(57,9 \%)$ e I6 homens $(42,1 \%)$, conforme gráfico I.

Gráfico I - Estado nutricional dos adultos (30-6o anos de idade) conforme o sexo.

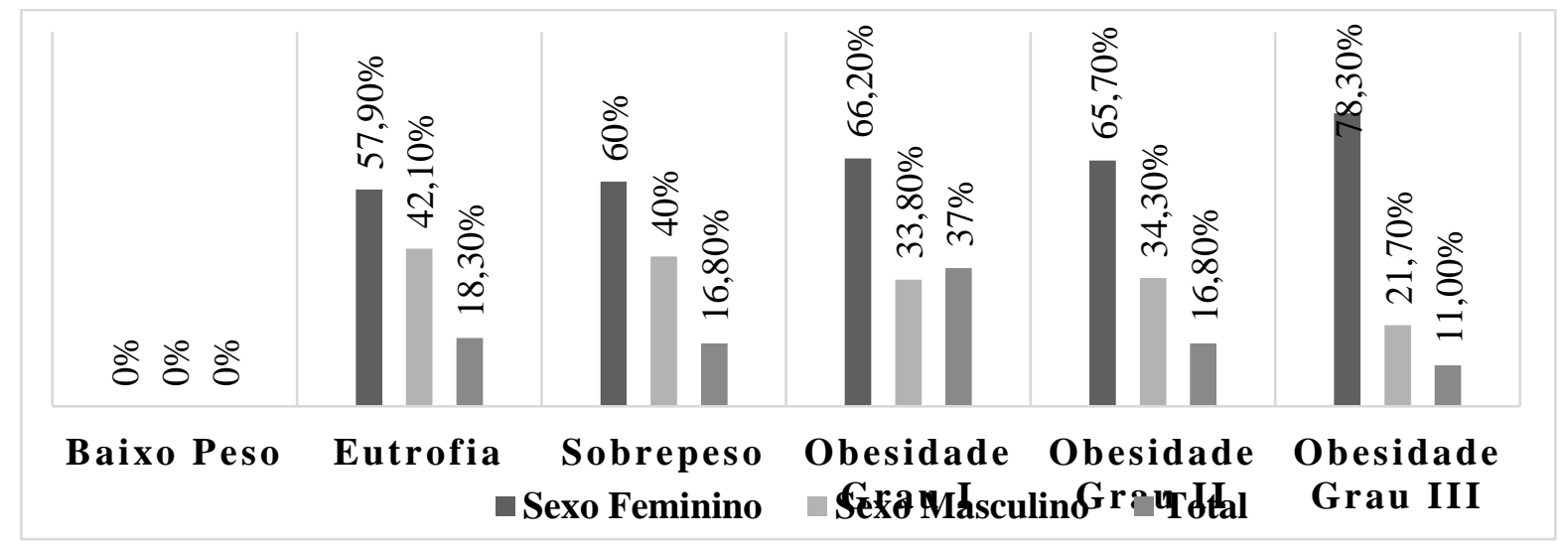

Fonte: as autoras.

Já na população idosa, conforme a classificação do índice de massa corporal, em que o IMC $\leq 22$ considera-se "baixo peso", >22 e < 27 "adequado ou eutrófico" e $\geq 27$ "sobrepeso" (BRASIL, 20II), a maior parte dos idosos deste estudo estavam em eutrofia, sendo um total de $37(48,7 \%)$ indivíduos, em que 25 eram mulheres $(67,5 \%)$ e 12 eram homens $(32,4 \%)$. Porém, o sobrepeso segue a eutrofia nesta amostra, em 35 pessoas (46\%), sendo 22 mulheres $(62,8 \%)$ e 13 homens $(37,1 \%)$. Apenas 4 indivíduos do total estudado apresentaram baixo peso (2 mulheres e 2 homens), qual revela o Gráfico 2.

Gráfico 2 - Estado nutricional dos idosos (>6o anos de idade) conforme o sexo.

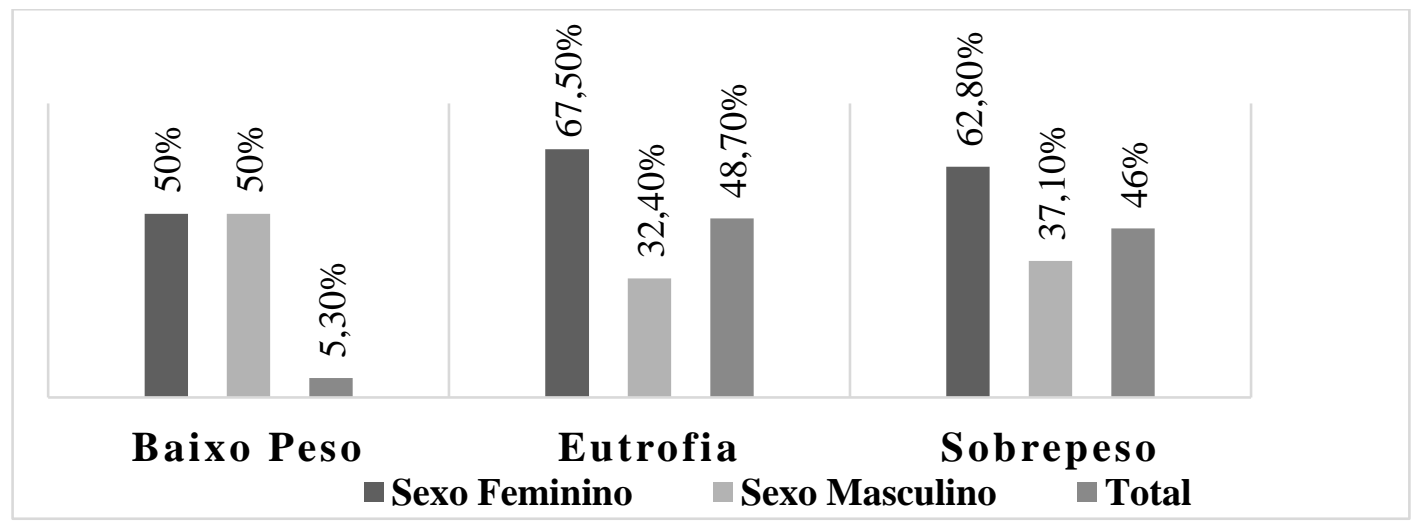

Fonte: as autoras 
A obesidade, caracterizada como o estado mais grave do excesso de peso, é uma das doenças que integra o grupo de doenças e agravos não transmissíveis (CORDEIRO; FREITAS, 2016), e um dos principais problemas da saúde pública em vários países, principalmente os que estão em desenvolvimento; é mencionada pela Organização Mundial de Saúde (OMS), como uma epidemia mundial. Esta, é definida como excesso de gordura no corpo, devido ao seu acúmulo, caracterizada por um IMC igual ou acima de 30 para adultos, contudo, o ideal é que o IMC seja utilizado junto de outros métodos de determinação de gordura corporal, devido seu uso apresentar limitações constatadas na literatura (ABESO, 2016).

A obesidade é associada a riscos para a saúde que envolve fatores biológicos, comportamentais e sociais. Seu aumento expressivo estabelece um dos motivos para explicar o aumento das doenças crônicas não-transmissíveis (DCNT), sendo estas, um dos principais fatores de risco para diversos agravos, como diabetes tipo II, doenças cardiovasculares, câncer (ALMEIDA et al., 2017), dislipidemias, distúrbios psicossociais, osteoartrites e hipertensão (CORDEIRO; FREITAS, 20I6).

A tabela abaixo (tabela 3 ), demonstra as principais patologias que acometem a população estudada, conforme o sexo.

Tabela 3 - Patologias apresentadas pela amostra conforme o sexo.

\begin{tabular}{cccc}
\hline Patologias & $\begin{array}{c}\text { Feminino } \\
(\mathrm{n}=\mathrm{I84})\end{array}$ & $\begin{array}{c}\text { Masculino } \\
(\mathrm{n}=\mathrm{I00})\end{array}$ & $\begin{array}{c}\text { Total } \\
(\mathrm{n}=\mathbf{2 8 4})\end{array}$ \\
\hline Obesidade & 40 & 20 & 60 \\
\hline Obesidade/ DM* 2 & I9 & 4 & 23 \\
\hline Obesidade/ HAS* & 4 & 8 & 12 \\
\hline Obesidade/DM2/HAS & 6 & 6 & I2 \\
\hline Obesidade/ Pré-diabetes/HAS & I & I & I5 \\
\hline Obesidade/Dislipidemia & I2 & 3 & 12 \\
\hline Obesidade/DM2/Dislipidemia & II & I & 34 \\
\hline Dislipidemia & I9 & I5 & 4 \\
\hline Dislipidemia/HAS & 3 & I & \\
\hline
\end{tabular}




\begin{tabular}{cccc}
\hline Pré-diabetes & 8 & 4 & 12 \\
\hline DM2* $^{*}$ & 24 & I5 & 39 \\
\hline HAS/ DM2 & 4 & I & 5 \\
\hline Gastrite & 3 & 2 & 5 \\
\hline Depressão & 3 & I & 4
\end{tabular}

Fonte: as autoras.

*Diabetes Mellitus tipo 2

** Hipertensão Arterial Sistêmica

Conforme observado na tabela acima, 6o (21,1\%) indivíduos da amostra total apresentaram obesidade; em que $40(66,7 \%)$ eram mulheres e 20 (33,3\%) eram homens; seguindo de diabetes mellitus tipo 2, em 39 (13,7\%) usuários, sendo 24 mulheres (61,5\%) e is homens $(38,5 \%)$; e dislipidemia em 34 pessoas (I2\%), sendo ig mulheres $(55,9 \%)$ e is homens $(44,12 \%)$.

Ressalta-se que do total da população estudada, dois (2) indivíduos do sexo feminino apresentaram obesidade, dislipidemia e HAS; dois (2) obesidade, DM, HAS, dislipidemia e cardiopatia; dois (2) obesidade, pré-DM, HAS e hipotireoidismo e dois (2), compulsão alimentar. Também verificou-se que um (I) indivíduo do sexo feminino tem como patologias obesidade, DM, HAS e dislipidemia; um (I) obesidade e anemia; um (I) obesidade e compulsão alimentar; um (I) dislipidemia e cardiopatia; um (I) dislipidemia, HAS e hipotireoidismo; um (I) enfisema; um (I) DM e esteatose hepática; um (I) estenose e estreitamento do esôfago; um (I) nefropatia; um (I) AVC (Acidente Vascular Cerebral) e um (I) lúpus eritematoso sistêmico.

Além do mais, os resultados do estudo mostraram em indivíduos do sexo masculino o seguinte: um (I) indivíduo apresentou obesidade, DM e compulsão alimentar; um (I) obesidade, dislipidemia, pré-DM e hipotireoidismo; um (I) obesidade, esteatose hepática e pré-DM; um (I) dislipidemia, pré-DM e HAS; um (I) dislipidemia e esteatose hepática; um (I) dislipidemia e gota; um (I) hipotireoidismo, DM e HAS; um (I) esteatose hepática; um (I) DM e cardiopatia e um (I) estenose e hemocromatose.

Finalmente, o estudo revelou que entre ambos os sexos, 3 indivíduos apresentaram dislipidemia e DM (sendo estes, 3 mulheres); três (3) dislipidemia, DM e HAS (sendo 2 
mulheres e I homem); três (3) dislipidemia e pré-DM (sendo os 3, homens); três (3) HAS (sendo também, 3 homens) e por fim três (3), câncer (sendo 2 mulheres e I homem).

Como mencionado anteriormente, a obesidade é a principal patologia entre ambos os sexos, contribuindo ao aparecimento de comorbidades associadas; o que pôde-se perceber neste estudo.

Segundo o Sistema de Vigilância de Fatores de Risco e Proteção para Doenças Crônicas por Inquérito Telefônico (VIGITEL), no Brasil, as estimativas de prevalência de obesidade subiram de 15 para $18 \%$ entre 2010 e 2014, em ambos os sexos (BRASIL, 2017).

O aumento da obesidade é explicado devido às mudanças comportamentais ocorridas nas últimas décadas, sendo as principais, a alimentação inadequada e o sedentarismo (FERREIRA; SZWARCWALD; DAMACENA, 2019). Isto implica na necessidade de novas políticas de promoção, prevenção e recuperação da saúde dos indivíduos (ALMEIDA et al., 2017).

Assim como a obesidade, o diabetes mellitus 2 e a dislipidemia também prevaleceram entre a população estudada. O Diabetes Mellitus (DM) é resultante da hiperglicemia causada por defeitos da secreção e/ou ação da insulina. É uma das quatro DCNT considerada como prioritária para intervenção da OMS e pelo Plano de Ações Estratégicas para o Enfrentamento das DCNT, 20II-2022. Devido suas inúmeras comorbidades, complicações e incapacidades, esta doença afeta a vida social e ocupacional dos acometidos, acarretando custos diretos e indiretos tanto aos portadores, sistemas de saúde quanto à sociedade (MALTA et al., 2019).

Estimativas apontam que entre os anos de 2010 e 2030 haverá o crescimento de cerca de 69\% do número de adultos com DM nos países em desenvolvimento e de $20 \%$ nos países desenvolvidos. Segundo dados da Pesquisa Nacional de Saúde, no Brasil, cerca de 9,2 milhões de brasileiros terão o diagnóstico de DM, sendo prevalente conforme o aumento da idade (SHAW; SICREE; ZIMMET, 20Iо).

Segundo a Sociedade Brasileira de Diabetes (SBD), para o controle do diabetes, é preciso desenvolver novas e mais fortes parcerias entre órgãos do governo e a sociedade civil, para uma maior cooperação em ações orientadas para prevenção, detecção e controle do diabetes (SBD, 2020). 
Em relação à dislipidemia, esta é caracterizada como um distúrbio no metabolismo das lipoproteínas, como o aumento do colesterol total, da lipoproteína de baixa densidade (LDL) e dos triglicerídeos, com a diminuição da lipoproteína de alta densidade (HDL); desenvolvida de acordo com fatores genéticos e/ou ambientais. Estas alterações dos lipídeos contribuem para o desenvolvimento de doenças como a doença arterial coronariana (DAC), hipertensão arterial sistêmica (HAS) e aterosclerose, sendo também secundárias à obesidade (SOUZA et al., 2019).

Estudos observacionais de base populacional conduzidos, revelaram a prevalência da dislipidemia acima de 60\% e 75\% em indivíduos obesos. Além disto, a Pesquisa Nacional de Saúde de 2013 apresentou uma prevalência de $12,5 \%$ na população com idade superior a I8 anos; e o inquérito da pesquisa de Vigilância de Fatores de Risco e Proteção para Doenças Crônicas por Inquérito Telefônico (VIGITEL) do ano de 20I6, encontrou o diagnóstico médico de dislipidemia de 24,8\%, ao investigar todas as capitais brasileiras (BRASIL, 2017).

Diante do que foi apresentado neste estudo, tornam-se essenciais ações de promoção da saúde, e prevenção de doenças na APS. Ressaltando-se a importância da abordagem multidisciplinar, sobretudo, pelo aspecto de integralidade aos cuidados de saúde. A adequação das equipes multidisciplinares é essencial para a $\mathrm{AB}$. Com a atuação de diferentes profissionais e áreas, a unidade tem como objetivo garantir o conceito ampliado de saúde, preconizado pelo SUS (LINHARES; ALBUQUERQUE; FERREIRA, 2020).

\section{CONCLUSÃO}

Os dados desta pesquisa permitiram concluir que as patologias mais frequentes na população adulta e idosa foram a obesidade, diabetes mellitus tipo 2 e dislipidemia; mas as mulheres acima de 40 anos foram as principais acometidas. Em relação ao estado nutricional, os adultos enquadraram-se em obesidade grau I, já os idosos, em sua maioria, estavam eutróficos.

Observou-se também que a obesidade é a porta de entrada para outras patologias como as quais, foram também apresentadas no trabalho. Devido ao alto custo para tratar estas doenças, o governo precisa de estratégias e intervenções especializadas na Atenção Básica, a fim de reduzir os gastos e tratar a obesidade e patologias associadas. 
Tendo em vista a grande relação destas patologias com estilo de vida, principalmente no que se refere a alimentação, neste estudo fica evidente também a importância do nutricionista na atenção básica à saúde. Este, tem papel fundamental na prevenção de doenças, principalmente naquelas que foram relatadas, orientando sobre a alimentação e promovendo o um controle do estado nutricional dos usuários. Salienta-se ainda a atuação do nutricionista como essencial em todas as fases da vida, visto que as condições de alimentação e nutrição constituem um dos direitos humanos fundamentais.

\section{REFERÊNCIAS}

ALMEIDA de, L. M., et al. Estratégias e desafios da gestão da atenção primária à saúde no controle e prevenção da obesidade. Revista Eletrônica Gestão \& Saúde, Brasília, v. 8, n. I, p. II4-139, jan. 2017. Disponível em:

〈https://periodicos.unb.br/index.php/rgs/article/view/3700〉. Acesso em: 23 mai. 202I.

ABESO- Associação Brasileira para o Estudo da Obesidade e da Síndrome Metabólica.

Diretrizes brasileiras de obesidade 2016 / ABESO - Associação Brasileira para o Estudo da Obesidade e da Síndrome Metabólica. - 4.ed. - São Paulo, SP. Disponível em: 〈https://abeso.org.br/diretrizes/>. Acesso em: 02 jul. 202I.

BRASIL. Ministério da Saúde. Secretaria de Atenção à Saúde. Departamento de Atenção Básica. Orientações para a coleta e análise de dados antropométricos em serviços de saúde: Norma Técnica do Sistema de Vigilância Alimentar e Nutricional - SISVAN / Ministério da Saúde, Secretaria de Atenção à Saúde, Departamento de Atenção Básica. Brasília: Ministério da Saúde, 20Ir. Disponível em: 〈https://bvsms.saude.gov.br/bvs/publicacoes/orientacoes_coleta_analise_dados_antropom etricos.pdf $>$. Acesso em: 30 jun. 2021.

BRASIL. Ministério da Saúde. Vigitel Brasil 2016 Saúde Suplementar: vigilância de fatores de risco e proteção para doenças crônicas por inquérito telefônico / Ministério da Saúde, Agência Nacional de Saúde Suplementar. - Brasília: Ministério da Saúde, 2017. 
Disponível em:

〈https://bvsms.saude.gov.br/bvs/publicacoes/vigitel_brasil_2or6_saude_suplementar.pdf〉. Acesso em: 27 jul. 2021.

CORDEIRO, J. Y. F.; FREITAS, S. R. S. Prevalência e fatores associados ao excesso de peso em uma população urbana do interior do amazonas, Brasil. Ver. Bras. Promoç. Saúde, Fortaleza, v. 29, n. 4, p. 533-543, out./dez. 2016. Disponível em:

$<$ https://periodicos.unifor.br/RBPS/article/view/5354\#: :text=Na\%20an\%C3\%Arlise\%20a justada\%2opara\%20a,p\%3Do\%2Coo)>. Acesso em: 23 mai. 2021.

FERREIRA, A. P. S.; SZWARCWALD, C. L.; DAMACENA, G. N. Prevalência e fatores associados da obesidade na população brasileira: estudo com dados aferidos da Pesquisa Nacional de Saúde, 2013. Rev. bras. epidemiol, [S.1], [S.v], [S.n], 2019. Disponível em:

〈https://www.scielo.br/j/rbepid/a/ZqgTbxFkLrQByhDq5Z5tXcG/abstract/?lang=pt〉. Acesso em: 25 mai. 2021.

FERREIRA, V. H. S., et al. Caracterização dos usuários de serviços de saúde de atenção primária em municípios paraibanos. In: CONGRESSO BRASILEIRO DE CIÊNCIAS DA SAÚDE, 2., 2017, Campina Grande. Anais [...]. Campina Grande, 2017. Disponível em:

〈https://www.editorarealize.com.br/editora/anais/conbracis/2017/TRABALHO_EVo7I_ MD4_SA7_ID2335_I5052017212845.pdf>. Acesso em: 27 mai. 2021.

LINHARES, A. M. R. L. C.; AlBUQUERQUE, R. A. S.; FERREIRA, F. V. Atuação do nutricionista na estratégia saúde da família: uma revisão integrativa. Revista Desafios, Tocantins, v. 7, n. 3, 2020. Disponível em: 〈https://sistemas.uft.edu.br/periodicos/index.php/desafios/article/view/6503/r7352〉. Acesso em: I8 jun. 2020. 
MALTA, D. C., et al. Prevalência de diabetes mellitus determinada pela hemoglobina glicada na população adulta brasileira, Pesquisa Nacional de Saúde. Rev. bras. epidemiol, [S.1], [S.v], [S.n], 2019. Disponível em: 〈https://www.scielo.br/j/rbepid/a/qQttB6XwmqzJYgcZKfpMV7L/?format=pdf\&lang= pt>. Acesso em: 26 mai. 202I.

PIMENTEL, Í. R. S., et al. Caracterização da demanda em uma Unidade de Saúde da Família. Rev bras med fam comunidade, Florianópolis, v. 6, n. 20, p. 175-18I, jul./set. 2011. Disponível em: 〈https://www.rbmfc.org.br/rbmfc/article/view/95/364〉. Acesso em: 22 mai. 202I.

SHAW, J.E; SICREE, R.A; ZIMMET, P.Z. Global estimates of the prevalence of diabetes for 2010 and 2030. Diabetes Res Clin Pract, Australia, v. 87, n. I, p. 4-14, oct./nov. 2010. Available in: < https://www.diabetesresearchclinicalpractice.com/article/Sor688227(09)00432-X/fulltext>. Access on July 29. 2021.

\section{SOCIEDADE BRASILEIRA DE DIABETES. Diretrizes da Sociedade Brasileira de}

Diabetes 2019-2020. São Paulo: Clannad; 2019 [acessado em Io mai. 2021]. Disponível em: <https://www.diabetes.org.br/profissionais/images/DIRETRIZES-COMPLETA-2or92020.pdf >. Acesso em: I7 jun. 2021.

SOUZA, N. A., et al. Dislipidemia familiar e fatores associados a alterações no perfil lipídico em crianças. Ciênc. saúde colet., Rio de Janeiro, v. 24, n. I, jan. 2019. Disponível em: 〈https://www.scielosp.org/article/csc/2019.v24nI/323-332/〉. Acesso em: 17 jun. 2021. 